\title{
First Measurement of Muon Neutrino Charged Current Quasielastic (CCQE) Double Differential Cross Section
}

\author{
Teppei Katori for the MiniBooNE collaboration
}

Massachusetts Institute of Technology, Cambridge, $\mathrm{MA}^{1}$

\begin{abstract}
Using a high statistics sample of muon neutrino charged current quasielastic (CCQE) events, we report the first measurement of the double differential cross section $\left(\frac{d^{2} \sigma}{d T_{\mu} d \cos \theta_{\mu}}\right)$ for this process. The result features reduced model dependence and supplies the most complete information on neutrino CCQE scattering to date. Measurements of the absolute cross section as a function of neutrino energy $\left(\sigma\left[E_{v}^{Q E, R F G}\right]\right)$ and the single differential cross section $\left(\frac{d \sigma}{d Q_{Q E}^{2}}\right)$ are also provided, largely to facilitate comparison with prior measurements. This data is of particular use for understanding the axial-vector form factor of the nucleon as well as improving the simulation of low energy neutrino interactions on nuclear targets, which is of particular relevance for experiments searching for neutrino oscillations.
\end{abstract}

Keywords: axial mass, charged current quasi-elastic, neutrino, MiniBooNE, cross section PACS: $11.80 . \mathrm{Cr}, 13.15 .+\mathrm{g}, 14.60 . \mathrm{Lm}, 14.60 . \mathrm{Pq}$

\section{CCQE EVENT SELECTION IN MINIBOONE}

The MiniBooNE2 detector, a spherical tank filled with mineral oil, is surrounded by 12808 " photomultiplier tubes (PMTs) to detect Čerenkov light from charged particles 3 . In the $19.2 \mu \mathrm{s}$ readout window, a "subevent" is defined as a timing cluster of PMT hits. The identification of $v_{\mu}$ CCQE interactions relies solely on the detection of the primary muon Čerenkov light (first subevent) and the associated decay electron Čerenkov light (second subevent) in these events [4]:

$$
\begin{array}{lll}
1: & v_{\mu}+n \rightarrow & \mu^{-}+p \\
2: & & \mu^{-} \rightarrow e^{-}+\bar{v}_{e}+v_{\mu} .
\end{array}
$$

where each line in this equation identifies the subevent where each process occurs. Therefore, a CCQE candidate is characterized with a total of 2 subevents. After cuts, 146070 events are identified from $5.58 \times 10^{20}$ protons on target collected between August 2002 and December 2005. The cuts are estimated to be $26 \%$ efficient at selecting $v_{\mu}$ CCQE events in a $550 \mathrm{~cm}$ radius, with a CCQE purity of $78 \%$.

The largest background is that from CC single-pion production $\left(\mathrm{CC} 1 \pi^{+}\right)$. The $\mathrm{CC} 1 \pi^{+}$interaction, proceeds as,

$$
\begin{array}{lll}
1: & v_{\mu}+p(n) \rightarrow & \mu^{-}+p(n)+\pi^{+}, \pi^{+} \rightarrow \mu^{+}+v_{\mu} \\
2: & & \mu^{-} \rightarrow e^{-}+\bar{v}_{e}+v_{\mu} \\
3: & & \mu^{+} \rightarrow e^{+}+v_{e}+\bar{v}_{\mu} .
\end{array}
$$

Note this interaction results in total 3 subevents, the primary interaction and 2 muon decays resulting in an electron and a positron. Although these events can be removed from the CCQE sample by requiring only one muon decay (a total of 2 subevents), there is still a significant number of $\mathrm{CC} 1 \pi^{+}$events that contribute to the CCQE background because one of the muon decays may be missed for various reasons. Among them, $\pi^{+}$absorption is a large effect (>40\%) with large uncertainty $(\sim 30 \%)$. Additionally, the prediction of $\mathrm{CC} 1 \pi^{+}$backgrounds in the CCQE sample rely on the Rein and Sehgal's model [5] and final state interactions (FSIs) in the NUANCE event generator [6] which are not sufficiently accurate for a precise background prediction to measure the absolute CCQE cross section.

\footnotetext{
$1 \mathrm{Ph} . \mathrm{D}$ thesis work at Indiana University, Bloomington, IN

2 The mini-Booster neutrino experiment (MiniBooNE) at Fermi National Accelerator Laboratory (Fermilab) is designed to search for $v_{\mu} \rightarrow v_{e}$ appearance neutrino oscillations [1].

${ }^{3}$ Detailed information about the Fermilab Booster neutrino beamline and the MiniBooNE neutrino detector are available elsewhere [2, 3].
} 

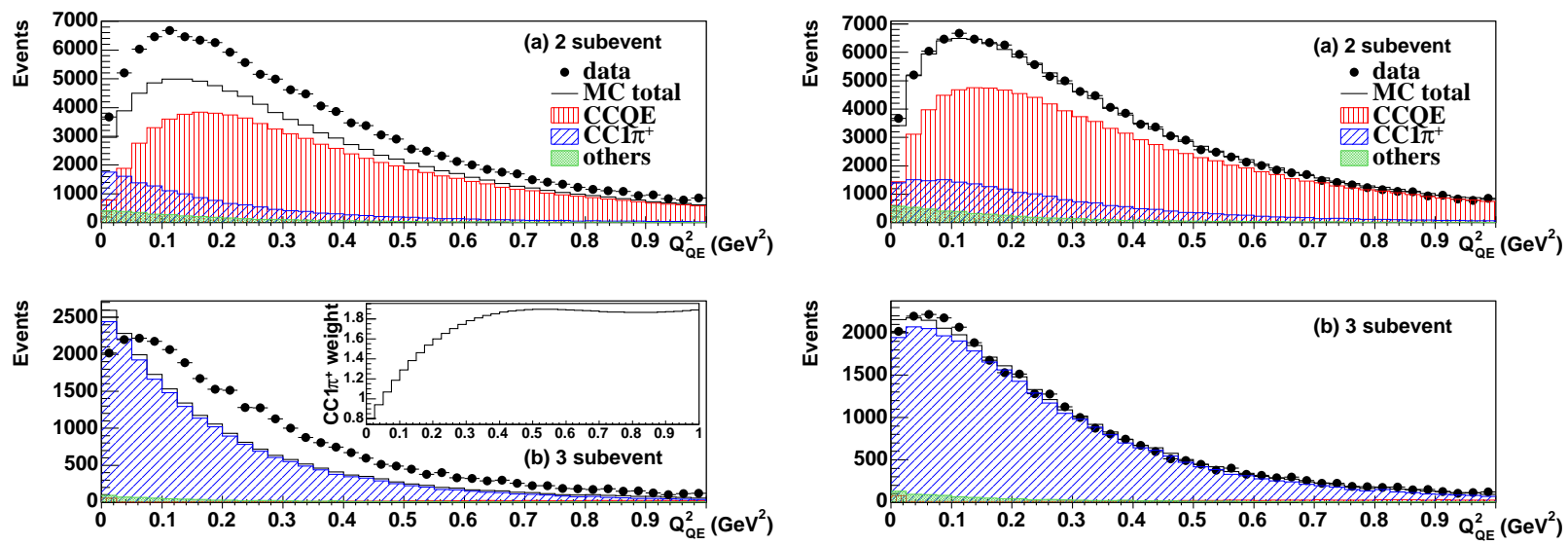

FIGURE 1. (color online). The distribution of events in $Q_{Q E}^{2}$ for the (a) 2 and (b) 3 subevent samples. Data and MC samples are shown along with the individual MC contributions from CCQE, CC1 $\pi^{+}$, and other channels. The left side is before the application of the $\mathrm{CC} 1 \pi^{+}$background correction. The inset in (b) shows the $\mathrm{CC} 1 \pi^{+}$reweighting function as determined from the background fit procedure. The right side is the same distribution after the application of the $\mathrm{CC} 1 \pi^{+}$background correction and the new $\mathrm{CCQE}$ model parameters $M_{A}^{e f f}$ and $\kappa$ as determined from the fit procedure described in the text.

\section{$\operatorname{CC} 1 \pi^{+}$BACKGROUND MEASUREMENT}

Because of uncertainties in the $\mathrm{CC} 1 \pi^{+}$background predictions, we instead measure the $\mathrm{CC} 1 \pi^{+}$rate in our $\mathrm{CC} 1 \pi^{+}$ data and the event generator is adjusted to match. By this, the predicted kinematic distribution of $\mathrm{CC} 1 \pi^{+}$events is modified, and the systematic error of $\mathrm{CC} 1 \pi^{+}$cross section is reduced to the level of the $\pi^{+}$absorption uncertainty.

The left plot in of Figure 1 shows the $Q_{Q E}^{2}$ distribution 4 for data and Monte Carlo (MC) of the two samples before the reweighting of $\mathrm{CC} 1 \pi^{+} \mathrm{MC}$ events. The 2-subevent sample shows good shape agreement between data and MC. NUANCE uses the relativistic Fermi gas (RFG) model [7] for CCQE interactions. In the previous work, we adjusted 2 parameters in RFG model, the effective axial mass $M_{A}^{e f f}$ and Pauli blocking parameter $\kappa$, to match the shape of the $Q_{Q E}^{2}$ distribution to data [4]. Note that analysis did not consider the overall normalization of events. The 3 -subevent sample shows a large data-MC disagreement in both shape and normalization. Using these samples, a simultaneous fit was performed for the shape and normalization of the 3-subevent sample, and the normalization of the 2-subevent sample. These were then used to determine the $\mathrm{CC} 1 \pi^{+}$reweighting function which is shown in the inset plot of Figure $1 \mathrm{~b}$ (left). In order to reduce the sensitivity to the details of the shape of the 2-subevent sample, only the $0.2<Q_{Q E}^{2}\left(\mathrm{GeV}^{2}\right)<0.6$ region was considered for the normalization parameter of this function. The $Q_{Q E}^{2}$ shape of the CCQE sample was fit later although it has no impact on the cross section measurements. The effect of the CCQE normalization on the 3-subevent sample was minimal since the background from CCQE in this $Q_{Q E}^{2}$ region is small as can be seen in the left plot of Figure $1 \mathrm{~b}$. As a final step, with the measured $\mathrm{CC} 1 \pi^{+}$background incorporated, a shape-only fit to the 2-subevent (CCQE) sample is performed in order to extract revised CCQE model parameters [4]. The normalization of the CCQE sample is then extracted from the fit described above. The $Q_{O E}^{2}$ distributions of data from all subevent samples is shown together with the MC prediction in the right plot of Figure 1. Data-MC agreement is good in both subevent samples. A fit to the 2-subevent sample provided adjusted CCQE model parameters, $M_{A}^{\text {eff }}$ and $\kappa$. This was a "shape-only" fit, that is, the MC was normalized with an arbitrary factor to have the same integrated event count as the background subtracted data. The fit yielded,

$$
\begin{aligned}
M_{A}^{e f f} & =1.35 \pm 0.17 \mathrm{GeV} / \mathrm{c}^{2} \\
\kappa & =1.007 \pm 0.012 \\
\chi^{2} / d o f & =47.0 / 38
\end{aligned}
$$

\footnotetext{
${ }^{4}$ The neutrino energy $E_{v}^{Q E}$ and 4-momentum transfer $Q_{Q E}^{2}$ are reconstructed by assuming a CCQE interaction and neutron at rest, with averaged nucleon binding energy $=34 \mathrm{MeV}$.
} 

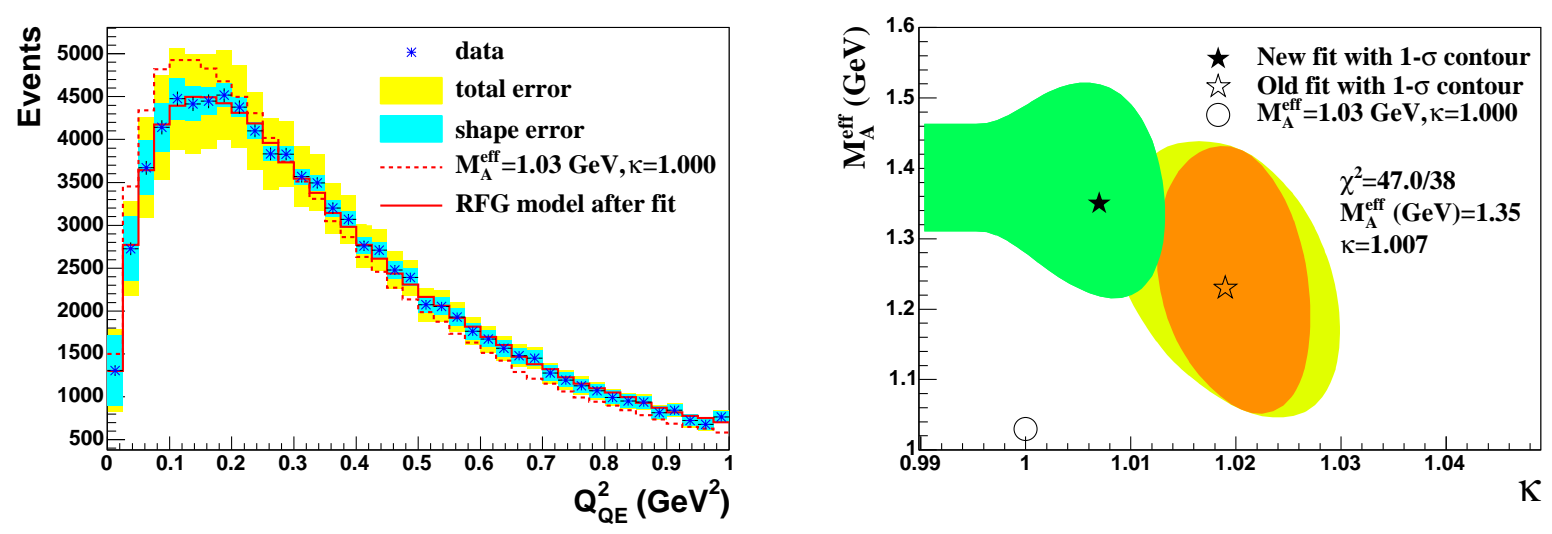

FIGURE 2. (Color online). Left plot is the $Q_{Q E}^{2}$ distribution of the data, MC before, and MC after the fit with errors. Right plot is the $1-\sigma$ contour plot for the $M_{A}^{e f f}-\kappa$ fit. The filled star shows the best fit point and $1-\sigma$ contour extracted from this work. The open star indicates the best fit point and $1-\sigma$ contour from the previous work [4]. Two regions are shown from the previous work, the larger area indicates the total uncertainty on the results including the background uncertainty [4].

The left plot of Figure 2 shows the $Q_{O E}^{2}$ distribution of data, MC before, and MC after the fit with all sources of error. Data and MC after the fit agree within shape errors. The right plot of Fig. 2 is the $1-\sigma$ contour regions of this fit together with the results from the previous MiniBooNE analysis [4]. Note that the current result is consistent (to within $1-\sigma$ ) with $\kappa=1$. This is because the $\mathrm{CC} 1 \pi^{+}$background resulting from the procedure in this work has changed by an amount only just consistent with the error assigned on the background in the previous work. The value for $\kappa$ is quite sensitive to the $\mathrm{CC} 1 \pi^{+}$background at low $Q_{Q E}^{2}$. However, the previous and current results are consistent at the $1-\sigma$ level.

The effect of the new $M_{A}^{e f f}$ is clearly seen in 2-dimensional plots. Figure 3 shows the data-MC ratio of CCQE candidate events as a function of muon kinetic energy $T_{\mu}(\mathrm{GeV})$ and muon scattering angle $\cos _{\mu}$. Note the muon energy and muon scattering angle observables are the basis of all reconstructed kinematics variables in the $v_{\mu}$ CCQE channel in MiniBooNE. In the left plot, we use the world averaged nuclear parameters $\left(M_{A}^{e f f}=1.03 \mathrm{GeV} / \mathrm{c}^{2}, \kappa=1.000\right)[8]$. As can be seen, data-MC disagreement follows auxiliary lines of equal $Q^{2}$. This is the same tendency observed in the previous CCQE analysis in MiniBooNE [4], indicating that data-MC disagreement is more likely due to an incorrect cross section prediction (=function of $Q^{2}$ ) than an incorrect flux prediction (=function of neutrino energy). After introducing the new $M_{A}^{e f f}$ and $\kappa\left(M_{A}^{e f f}=1.35 \mathrm{GeV} / \mathrm{c}^{2}, \kappa=1.007\right)$, Fig. 3 right plot, data-MC disagreement is reduced and we obtain an improved cross section prediction across the entire kinematic space.

Note, this modification of the CCQE cross section prediction does not affect the CCQE absolute cross section measurement, presented below.

\section{CCQE ABSOLUTE CROSS SECTION MEASUREMENTS}

\section{Flux-averaged double differential cross section}

Figure. 4 shows the flux-averaged double differential cross section, $\frac{d^{2} \sigma}{d T_{\mu} d \cos \mu}$, for the $v_{\mu}$ CCQE process. The fluxaveraged total cross section, an integral of the double differential cross section $\left(-1<\cos _{\mu}<+1\right.$ and $0<T_{\mu}(\mathrm{GeV})<$ $\infty)$ is $9.412 \times 10^{-39} \mathrm{~cm}^{2}$. The total normalization error on this measurement is $10.8 \%$.

The kinematic quantities, $T_{\mu}$ and $\cos _{\mu}$, have been corrected for detector resolution effects only. This result is the most model-independent measurement of this process possible with the MiniBooNE detector. No cuts on the recoil nucleons are used to define this process. The neutrino flux is an absolute prediction and was not adjusted based on measured processes in the MiniBooNE detector. 

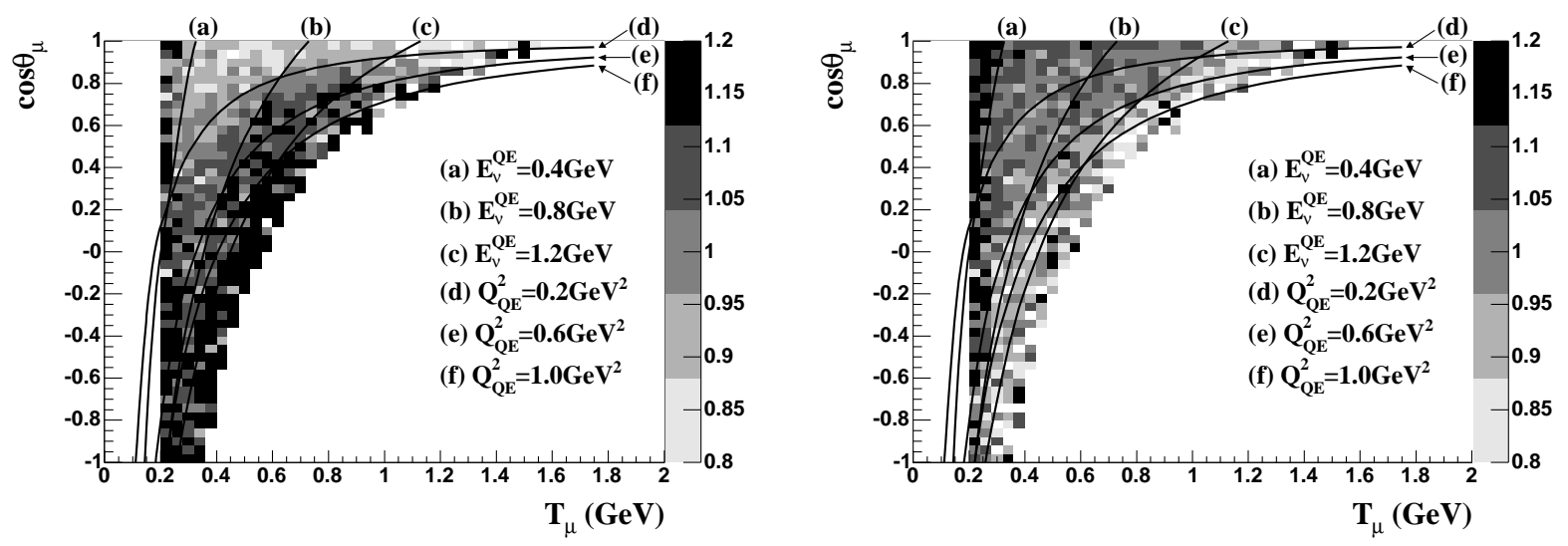

FIGURE 3. Ratio of MiniBooNE $v_{\mu}$ CCQE data/simulation as a function of measured muon angle and kinetic energy. Left plot, with world averaged $M_{A}^{e f f}\left(=1.03 \mathrm{GeV} / \mathrm{c}^{2}\right)$ and $\kappa(=1.000)$, and right plot, with newly determined $M_{A}^{\text {eff }}\left(=1.35 \mathrm{GeV} / \mathrm{c}^{2}\right)$ and $\kappa(=1.007)$. The ratio forms a $2 \mathrm{D}$ surface whose values are represented by the gray scale, shown on the right. If the simulation modeled the data perfectly, the ratio would be unity everywhere. Contours of constant $E_{v}$ and $Q^{2}$ are overlaid.

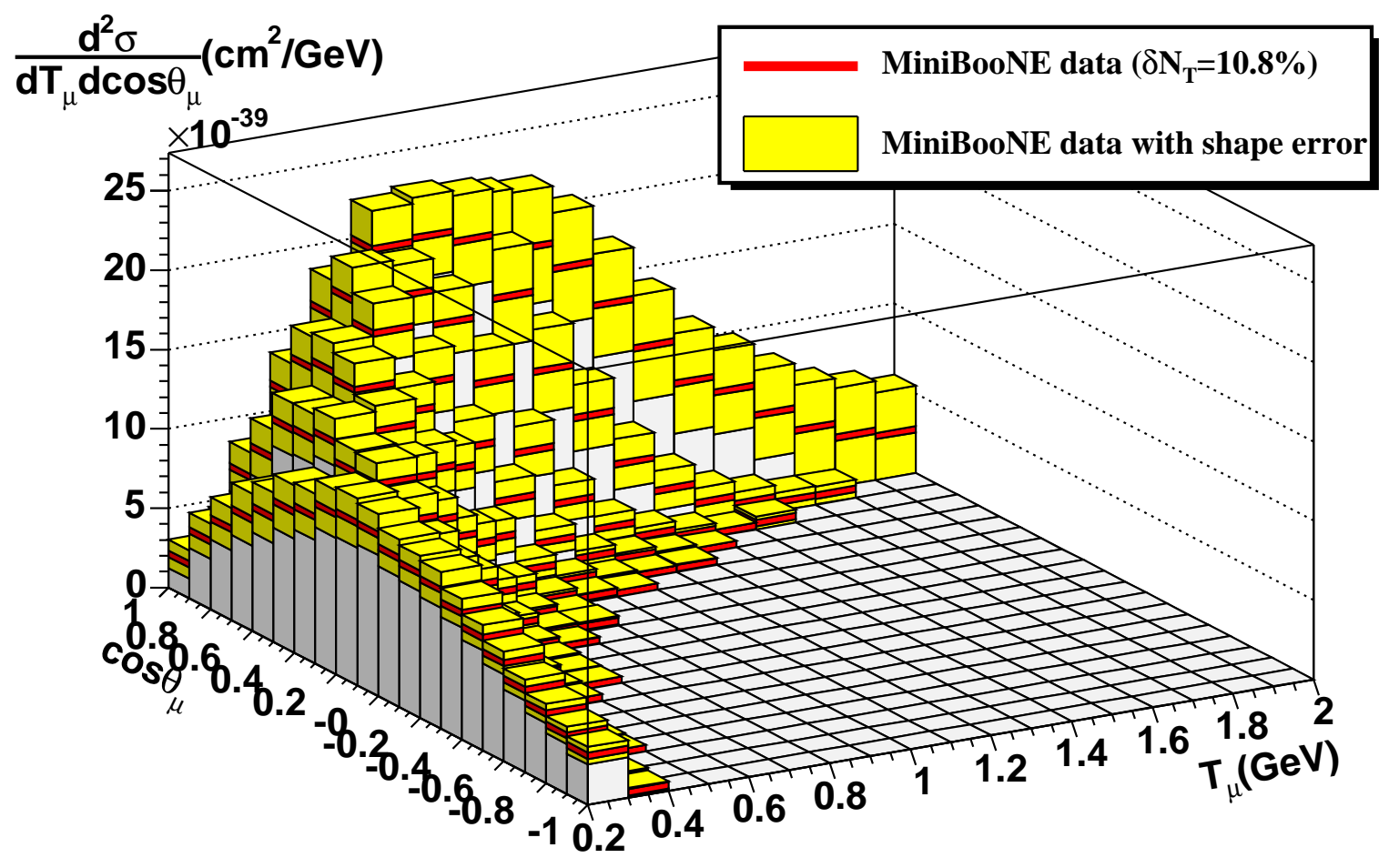

FIGURE 4. (Color online). The flux-averaged double differential per nucleon $(n)$ cross section for the $v_{\mu}$ CCQE process. The dark bars indicate the measured values and the surrounding lighter bands show the shape error. The overall normalization (scale) error is $10.8 \%$. 


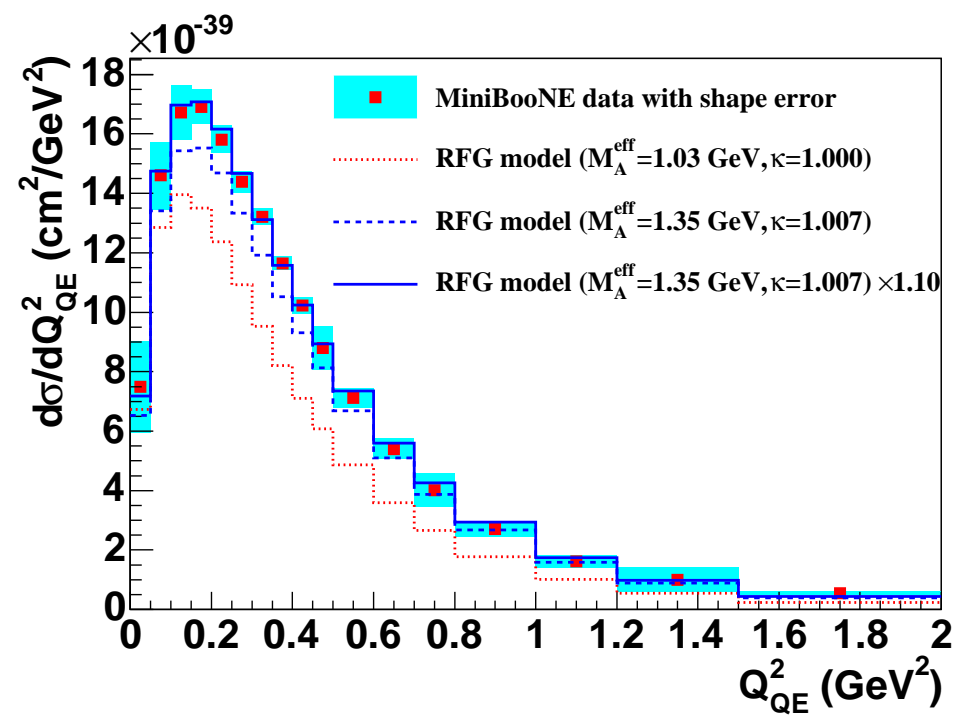

FIGURE 5. (Color online). The flux-averaged single differential per nucleon $(n)$ cross section for the $v_{\mu}$ CCQE process. The measured values are shown as points with the shape error as shaded bars. Predictions from the NUANCE RFG model with different values for the model parameters are shown as histograms.

\section{Flux-averaged differential cross section}

Figure. 5 shows the flux-averaged single differential cross section, $\frac{d \sigma}{d Q_{Q E}^{2}}$. The reconstructed 4-momentum transfer $Q_{Q E}^{2}$ depends only upon the (unfolded) quantities $T_{\mu}$ and $\cos _{\mu}$.

In addition to the experimental result, Figure 5 also shows the prediction for the CCQE process from the NUANCE simulation with three different variations of parameters in the underlying RFG model. The predictions are fluxaveraged and absolutely normalized. The RFG model is plotted with both the world-averaged CCQE parameters $\left(M_{A}=1.03 \mathrm{GeV}, \kappa=1.000\right)$ and with the CCQE parameters extracted from this analysis $\left(M_{A}=1.35 \mathrm{GeV}, \kappa=1.007\right)$. The model using the world-averaged CCQE parameters underpredicts the measured values significantly (by $\approx 30 \%$ ). The model using the CCQE parameters extracted from the shape fit to the MiniBooNE CCQE data are within $\approx 10 \%$ of the data, consistent within the normalization uncertainty of $\approx 10 \%$. The prediction with the CCQE parameters from this analysis scaled by 1.10 is also plotted and is in good agreement with the data.

\section{Flux-unfolded total cross section}

The flux-unfolded total cross section $\left(\sigma\left[E_{V}^{Q E, R F G}\right]\right)$ as a function of estimated neutrino energy $E_{V}^{Q E, R F G}$ is shown in Figure 6. The quantity $E_{v}^{Q E, R F G}$ is a model-dependent estimate of the neutrino energy obtained after correcting for both detector and nuclear model resolution effects. These results depend on the details of the nuclear model used for the calculation. The dependence is only weak in the peak of the flux distribution but becomes strong at $E_{v}<0.5 \mathrm{GeV}$ and $E_{v}>1.0 \mathrm{GeV}$, in the "tails" of the flux distribution.

In Figure 6 data are compared with the NUANCE implementation of the RFG model with the world averaged parameter values $\left(M_{A}^{e f f}=1.03 \mathrm{GeV}, \kappa=1.000\right)$, and the parameters extracted from this work $\left(M_{A}^{e f f}=1.35 \mathrm{GeV}\right.$, $\kappa=1.007)$. These are absolute predictions from the model - they are not scaled in any way. The measurement is $\sim 20 \%$ higher than the RFG model prediction with world average parameter values at the flux peak $(700-800 \mathrm{MeV})$. The prediction with the RFG parameter values extracted from the shape-only fit to MiniBooNE CCQE data reproduces the data significantly better, to within $1 \sigma$ for every point over the entire measured energy range.

Figure 6(b) shows the CCQE results from the LSND [9] and NOMAD [10] experiments. It is interesting to note that NOMAD results are better described with the world-average $M_{A}^{\text {eff }}$ and $\kappa$ values. 

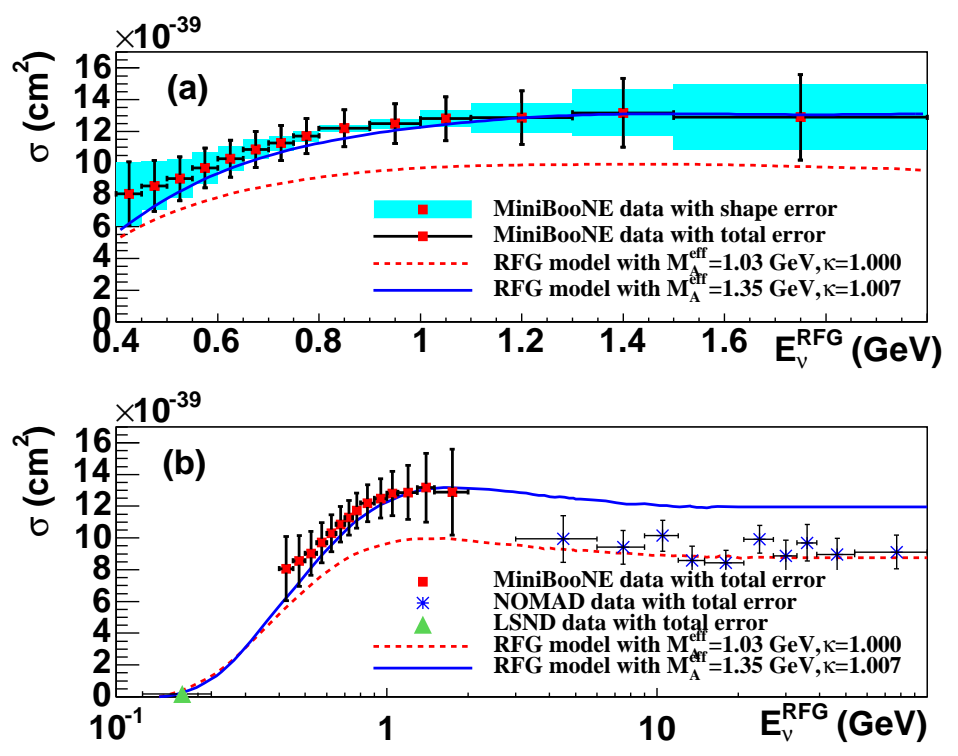

FIGURE 6. (Color online). The flux-unfolded total per nucleon (n) cross section with total errors and bin widths plotted indicated with the data points. In (a) shape errors are shown as shaded boxes. In (b), a larger energy range is shown along with results from the LSND [9] and NOMAD [10] experiments. Predictions from the NUANCE simulation with two different RFG parameter variations are shown in both plots.

At this time, a solution to this growing mystery is not evident. Although there are tremendous efforts to model this process [11], no models seem to be able to produce the (1) large observed $M_{A}^{\text {eff }}$ and (2) large observed total cross section, while keeping the "bare" $M_{A}=1.03 \mathrm{GeV}$ (the world averaged value). Model-independent cross section results from the MINOS near detector [12], running with $E_{v} \sim 3 \mathrm{GeV}$ and near-future experiments such as MINERvA [13], running with $2<E_{v}<20 \mathrm{GeV}$ could help shed further light on this subject.

\section{REFERENCES}

1. A. A. Aguilar-Arevalo et al. [MiniBooNE Collaboration], Phys. Rev. Lett. 98, 231801 (2007); Phys. Rev. Lett. 102, 101802 (2009); arXiv:0904.1958 [hep-ex].

2. A. A. Aguilar-Arevalo et al. [MiniBooNE Collaboration], Phys. Rev. D79, 072002 (2009).

3. A. A. Aguilar-Arevalo et al. [MiniBooNE Collaboration], Nucl. Instr. Meth. A599,28 (2009).

4. A. A. Aguilar-Arevalo et al. [MiniBooNE Collaboration], Phys. Rev. Lett. 100, 032301 (2008); Teppei Katori, AIP Conf. Proc. 967, 123 (2007); FERMILAB-THESIS-2008-64

5. D. Rein and L. M. Sehgal, Nucl. Phys. B223, 29 (1983).

6. D. Casper, Nucl. Phys. Proc. Suppl. 112, 161 (2002)

7. R. A. Smith and E. J. Moniz, Nucl. Phys. B43, 605 (1972); erratum: ibid. B101, 547 (1975)

8. V. Bernard et al., J. Phys. G28, R1 (2002).

9. L. B. Auerbach et al. [LSND Collaboration], Phys. Rev. C 66, 015501 (2002).

10. K. S. Kuzmin, V. V. Lyubushkin, and V. A. Naumov, Eur. Phys. J. C 54, 517 (2008); V. V. Lyubushkin et al. [NOMAD Collaboration], arXiv:0812.4543 [hep-ex].

11. J. E. Amaro et al., Phys. Rev. C71, 015501 (2005); Phys. Rev. C75, 034613 (2007); T. Leitner et al., Phys. Rev. C73, 065502 (2006); Phys. Rev. C79, 065502 (2006); O. Benhar et al., Phys. Rev. D72, 053005 (2005); arXiv:0903.2329] [hep-ph]; A. Butkevich et al., Phys. Rev. C72, 025501 (2005); Phys. Rev. C76, 045502 (2007); Phys. Rev. C80, 014610 (2009);

S. K. Singh et al., arXiv:0808.2103 [nucl-th]; J. Nieves et al., Phys. Rev. C73, 025504 (2006); N. Jachowicz et al., Phys. Rev. C73, 024607 (2006); A. M. Ankowski et al., Phys. Rev. C77, 044311 (2008).

12. M. Dorman, FERMILAB-THESIS-2008-72; in these proceedings

13. David Boehnlein, AIP Conf. Proc. 967, 304 (2007); M. Kordosky, in these proceedings 